\title{
Análisis crítico de un artículo: Levofloxacino profiláctico en pacientes neutropénicos
}

Roberto Candia B ${ }^{1, a}$, Gabriel Rada G ${ }^{1,2}$.

Critically appraised article: Levofloxacin to Prevent Bacterial Infection in Patients with Cancer and Neutropenia.

N Engl J Med 2005; 353: 977-87.

Bucaneve G, Micozzi A, Menichetti F, Martino $P$, Dionisi $M S$, Martinelli $G$, Allione $B, D$ 'Antonio $D$, Buelli $M$, Nosari AM, Cilloni $D$, Zuffa $E$, Cantaffa $R$, Specchia $G$, Amadori S, Fabbiano $F$, Deliliers GL, Lauria F, Foa $R$, Del Favero A; Gruppo Italiano Malattie Ematologiche dell'Adulto (GIMEMA) Infection Program.

Background: The prophylactic use of fluoroquinolones in patients with cancer and neutropenia is controversial and is not a recommended intervention. Methods: We randomly assigned 760 consecutive adult patients with cancer in whom chemotherapy-induced neutropenia ( $<1000$ neutrophils per cubic millimeter) was expected to occur for more than seven days to receive either oral levofloxacin (500 mg daily) or placebo from the start of chemotherapy until the resolution of neutropenia. Patients were stratified according to their underlying disease (acute leukemia vs solid tumor or lymphoma). Results: An intention-to-treat analysis showed that fever was present for the duration of neutropenia in 65 percent of patients who received levofloxacin prophylaxis, as compared with 85 percent of those receiving placebo ( 243 of 375 vs 308 of 363; relative risk, 0.76; absolute difference in risk, -20 percent; 95 percent confidence interval, -26 to -14 percent; $\mathrm{P}=0.001$ ). The levofloxacin

${ }^{1}$ Unidad de Medicina Basada en Evidencia.

${ }^{2}$ Departamento de Medicina Interna, Pontificia Universidad Católica de Chile. Santiago, Chile.

Residente. 
group had a lower rate of microbiologically documented infections (absolute difference in risk, -17 percent; 95 percent confidence interval, -24 to -10 percent; $\mathrm{P}<0.001$ ), bacteremias (difference in risk, -16 percent; 95 percent confidence interval, -22 to -9 percent; $\mathrm{P}<0.001$ ), and single-agent gram-negative bacteremias (difference in risk, -7 percent; 95 percent confidence interval, -10 to -2 percent; $P<0.01$ ) than did the placebo group. Mortality and tolerability were similar in the two groups. The effects of prophylaxis were also similar between patients with acute leukemia and those with solid tumors or lymphoma. Conclusions: Prophylactic treatment with levofloxacin is an effective and well-tolerated way of preventing febrile episodes and other relevant infection-related outcomes in patients with cancer and profound and protracted neutropenia. The long-term effect of this intervention on microbial resistance in the community is not known.

\section{CONCLUSIÓN DE LOS REVISORES:}

La profilaxis con levofloxacina disminuye los episodios febriles y los costos asociados en pacientes con neutropenia de alto riesgo, pero sin demostrar disminución de la mortalidad.

\section{PREGUNTA CLINICA:}

En pacientes con neutropenia de alto riesgo, posterior a quimioterapia, el uso de levofloxacina profiláctico, ¿disminuye el riesgo de episodios febriles, infecciones y mortalidad?

\section{ConTEXTO:}

En hospitales de alta complejidad frecuentemente nos enfrentamos a pacientes con neutropenia, ya sea como forma de presentación de algunas neoplasias o secundaria a la terapia de las mismas. En la década de los 60 , el $70 \%$ de las muertes en estos pacientes se debía a infecciones, siendo el síntoma principal la fiebre ${ }^{1}$. Por esta razón, los episodios febriles en el contexto de una neutropenia son eventos considerados graves, asociados a costos elevados, tanto económicos como humanos, por lo que se han establecido diferentes estrategias para prevenir tal condición. Una de ellas es el uso profiláctico de antibióticos previo y/o durante la neutropenia, lo cual no ha sido recomendado hasta ahora por consensos nacionales ${ }^{2}$ ni internacionales ${ }^{3}$.

\section{CARActerísticas Del ESTUdio:}

Tipo de estudio: Randomizado. Pacientes: 738 adultos con leucemia, linfoma o tumores sólidos con alta probabilidad de desarrollar neutropenia de alto riesgo (definida como recuento $<1.000$ neutrófilos por más de 7 días) inducida por quimioterapia (QMT), Intervención: 375 pacientes recibieron levofloxacina $500 \mathrm{mg} /$ día, iniciado 1 a 3 días previo a QMT, hasta resolución de la neutropenia. Comparación: 363 pacientes recibieron placebo, administrado en forma similar a la intervención. Outcome Primario: Episodios febriles que requirieron terapia antibiótica empírica. Fiebre definida como temperatura axilar mayor a $38,5^{\circ} \mathrm{C}$ en una toma o $38^{\circ} \mathrm{C}$ en dos tomas separadas por $12 \mathrm{~h}$. Outcomes Secundarios: Número de infecciones documentadas clínica y microbiológicamente (cultivos tradicionales); sobrevida posterior a la resolución de la neutropenia. 
VALIDEZ:

Randomizado:

SI

Ocultamiento de secuencia de aleatorización:

SI

$99,7 \%$

SI*

Análisis con intención de tratar:

SI

SI

Pacientes

Tratantes

SI

SI

Adjudicadores de eventos

SI

Análisis de datos

Grupos similares respecto a variables pronósticas conocidas:

* Sólo el outcome primario (episodios febriles) se analizó por intención de tratar, en el resto se realizó análisis por protocolo.

REsUlTADOS:

\begin{tabular}{|c|c|c|c|c|c|}
\hline OUTCOME & $\begin{array}{l}\text { TEE } \\
\text { Levof }\end{array}$ & $\begin{array}{c}\text { TEC } \\
\text { Placebo }\end{array}$ & $\begin{array}{c}\text { RRA } \\
(95 \% \text { IC) }\end{array}$ & $\begin{array}{c}\mathrm{RRR} \\
(95 \% \mathrm{IC})\end{array}$ & $\begin{array}{c}\text { NNT } \\
(95 \% \text { IC) }\end{array}$ \\
\hline Episodios & $64,8 \%$ & $84,8 \%$ & 0,20 & $24 \%$ & 5 \\
\hline Febriles & & & $(0,261$ a 0,14$)$ & (16\% a 31\%) & (4 a 7) \\
\hline Mortalidad & $3 \%$ & $5 \%$ & $\begin{array}{c}0,023 \\
(0,051 \text { a }-0,005)\end{array}$ & $\begin{array}{c}46 \% \\
(-10 \% \text { a } 100 \%)\end{array}$ & $\begin{array}{c}43 \\
\text { (20 a infinito) }\end{array}$ \\
\hline Infección & $21,8 \%$ & $39 \%$ & 0,172 & $44 \%$ & 6 \\
\hline Microbiología (+) & & & $(0,24$ a 0,104$)$ & (27\% a $62 \%)$ & $(4$ a 10$)$ \\
\hline Fiebre origen & $34,5 \%$ & $37,5 \%$ & 0,03 & $8 \%$ & 33 \\
\hline Desconocido & & & $(0,102$ a $-0,042)$ & $(-11 \%$ a $27 \%)$ & (10 a infinito) \\
\hline $\begin{array}{l}\text { Infección } \\
\text { (clínica) }\end{array}$ & $8,8 \%$ & $9,8 \%$ & $\begin{array}{c}0,01 \\
(0,054 \mathrm{a}-0,034)\end{array}$ & $\begin{array}{c}10 \% \\
(-35 \% \text { a } 55 \%)\end{array}$ & $\begin{array}{c}100 \\
\text { (19 a infinito) }\end{array}$ \\
\hline
\end{tabular}

$\mathrm{TEE}=$ Tasa de eventos Grupo Experimental, $\mathrm{TEC}=$ Tasa de eventos Grupo Control, RRA $=$ Reducción de Riesgo Absoluto, RRR= Reducción de Riesgo Relativo, NNT= Número Necesario para Tratar, IC= Intervalo de confianza.

\section{COMENTARIOS Y APLICACIÓN PRÁCTICA:}

- Estudio de alta calidad metodológica, que cumple con la mayoría de los criterios de validez interna. Si bien el análisis por intención de tratar se llevó a cabo sólo parcialmente, el outcome principal (episodios febriles) se analizó de esta forma.

- El uso profiláctico de levofloxacina disminuye los episodios febriles en pacientes con neutropenia de alto riesgo ( $>7$ días), lo que se asocia a una disminución en la indicación de terapia antibiótica empírica.

- No se logra demostrar beneficio en términos de mortalidad. La tasa histórica de mortalidad por infección en pacientes neutropénicos es de aproximadamente 5\%, por lo tanto se requieren estudios de mayor tamaño muestral para demostrar beneficio 
significativo para este outcome, sin embargo, la disminución de eventos febriles y de infecciones microbiológicamente comprobadas son outcomes relevantes para el paciente, ya que se asocian a disminución de los costos y al tiempo de hospitalización.

- En el análisis por protocolo se pesquisó una disminución significativa de infecciones documentadas microbiológicamente, lo que no se acompañó de un aumento de la incidencia de fiebre de origen desconocido. Esto en parte confirma que tal reducción no se explica simplemente por una menor detección de infecciones en contexto de la profilaxis, si no que correspondería a una reducción real del evento.

- Una de las debilidades del estudio es que no fue diseñado para evaluar la aparición de resistencia antibiótica, que es uno de los potenciales efectos no deseados de la aplicación de esta alternativa terapéutica.

- Las normas actuales no recomiendan el uso de antibióticos profilácticos en pacientes neutropénicos, ya que los estudios previos que mostraban beneficio eran pequeños y de baja calidad metodológica. El presente estudio es de buena calidad metodológica, de mayor tamaño muestral y muestra beneficio clínico, por lo tanto hace necesario reevaluar el uso de antibióticos (en este caso quinolonas) para esta indicación. Esto es reforzado por la existencia de una revisión sistemática ${ }^{4}$ y otro estudio randomizado reciente ${ }^{5}$ que demostraron beneficio clínico en el mismo sentido. No existen estudios comparativos que evalúen cuál antibiótico es el más apropiado como profilaxis, siendo los más utilizados ciprofloxacina y, actualmente, levofloxacina. Desde el punto de vista microbiológico, la cobertura de levofloxacina es más amplia que ciprofloxacina, sobre todo en relación a cocáceas gram positivas. Esto podría ser importante, ya que si bien la profilaxis con ciprofloxacina se ha relacionado a una disminución de infecciones por gram negativos, también se ha asociado a una mayor prevalencia de infecciones por gram positivos ${ }^{2}$.

- En definitiva, el uso de antibióticos profilácticos en pacientes con neutropenia febril es una intervención fácil y de bajo costo, que muestra beneficio en outcomes relevantes tanto para el paciente como para las autoridades en términos de costos económicos, además de sugerir un beneficio en morbimortalidad dada la relevancia clínica de un episodio de neutropenia febril en un paciente con cáncer. Una de las debilidades del estudio es que no fue diseñado para evaluar la aparición de resistencia antibiótica, hecho que es ampliamente criticado por los expertos que rechazan el uso de profilaxis antibiótica en estos pacientes; sin embargo, considerando el beneficio clínico demostrado, tal evento pudiera ser un outcome de menor relevancia.

\section{REFERENCIAS}

1. SchimpF SC, SATteriee W, Young VM, Serpick A. Empinic therapy with carbenicillin and gentamicin for febrile patients with cancer and granulocytopenia. N Engl J Med 1971; 284: 1061.

2. Santolaya ME, Rabaglati R, Bidart T, Paya E, Guzmán AM, Moraies R et al. Consenso manejo racional del paciente con cáncer, neutropenia y fiebre. Rev Chil Infect 2005; 22 (Supl 2): S79-S113.

3. Hughes WT, Armstrong D, Bodey GP, Bow EJ, Brown AE, Caiandra T et al 2002 guidelines for the use of antimicrobial agents in neutropenic patients with cancer. Clin Infect Dis 2002; 34: 730-51.

4. ENGeis EA, LAU J, BARZA M. Efficacy of quinolone prophylaxis in neutropenic cancer patients: a metaanalysis. J Clin Oncol 1998; 16: 1179-87.

5. Culen M, Steven N, Buwngham L, Gaunt C, Hastings M, Simmonds P et al. Antibacterial Prophylaxis after Chemotherapy for Solid Tumors and Lymphomas. N Engl J Med 2005; 353: 988-98. 